Alma Mater Studiorum - Università di Bologna DEPARTMENT OF ECONOMICS

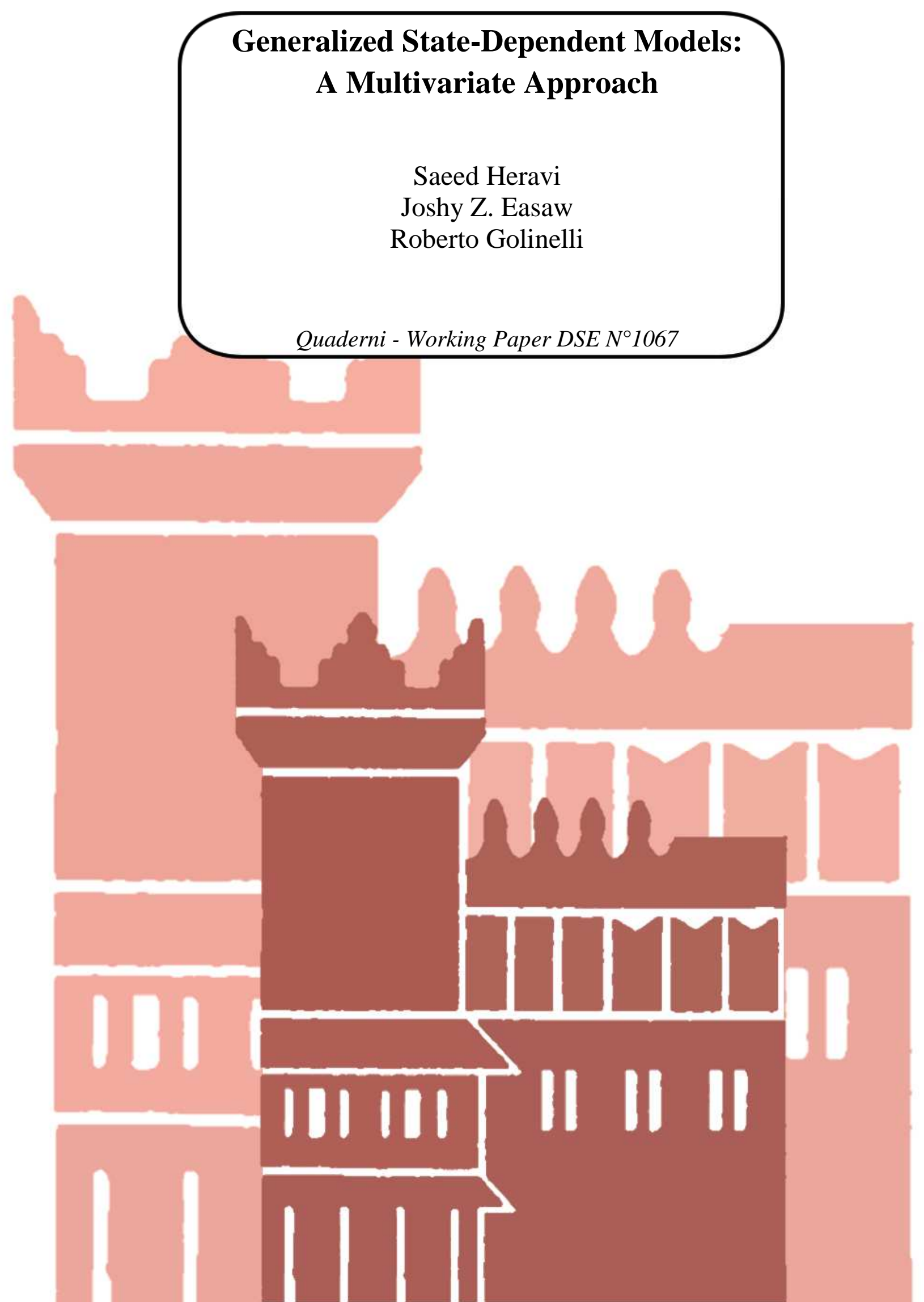




\title{
Generalized State-Dependent Models: A Multivariate Approach
}

\author{
Saeed Heravi ${ }^{a}$, Joshy Z. Easaw ${ }^{a}$ and Roberto Golinelli ${ }^{b}$ \\ ${ }^{a}$ Cardiff University Business School, Cardiff, UK \\ ${ }^{b}$ Department of Economics, University of Bologna, ITALY
}

$25^{\text {th }}$ May 2016

\begin{abstract}
The main purpose of this paper is to develop generalized 'State Dependent Models' (SDM) in a multivariate framework for empirical analysis. This significantly extends the existing SDM which only allow univariate analysis following a simple AR process. The extended model enables greater possibility for empirical analysis of economic relationships. The principle advantage of SDM is that it allows for a general form of non-linearity and can be fitted without any specific prior assumption about the form of non-linearity. We describe the general structure of the SDM and the problem of its identification is also considered. Finally, we apply the algorithm to show the impact of sentiment and income when modelling US consumption.
\end{abstract}

Keywords: Generalized State-Dependent Models, Multivariate Framework, Consumer Sentiments-Consumption Behavior

JEL: C32, C51, E32 


\section{I: Introduction}

The objectives of the paper are twofold. Firstly, we develop a generalized StateDependent Model (SDM) in a multivariate framework. Thereby, enabling a multivariate approach to analyzing a general form of non-linearity which can be fitted without any specific prior assumption about the form of non-linearity. Secondly, the extended framework enables greater applicability for the empirical analysis of economic relationships and models. The present paper provides an example by investigating empirically the relationship between households' income, sentiment and their consumption behavior. The paper significantly extends the existing literature on SDM and its applications where the focus has been on univariate analysis using a pure AR time series.

Priestley (1980) developed a general class of non-linear time series, called 'State Dependent Models' which includes non-linear time series models and linear ARMA as special cases. The principal advantage of SDM is that it allows for a general form of nonlinearity and can be fitted without any specific prior assumption about the form of nonlinearity. This usefully indicates the specific type of non-linear model that is appropriate to a particular relationship. Indeed, whether a linear model could be equally applicable.

The economic application is a topical one and lends itself neatly to the issue of the general form of non-linearity, which can be fitted without any specific prior assumptions. The role of the consumer sentiment both in predicting and understanding the causes of business cycles is an important one, and the existing literature has been largely analyzed in the linear context (with possible ad hoc shifts in parameters). However, it is also worth noting that consumer sentiment can be affected by sizeable shocks which, in turn, induce non-linear responses to the consumption behavior but is unaffected by small consumer sentiment shocks. . 
The paper is organised as follows. The next section outlines the extended generalized SDM which incorporates a multivariate framework. Section III considers the economic application of this extended SDM approach; notably the consumption-sentiment relationship. The analysis initially considers the standard linear approach and ad hoc non-linear extensions that are prevalent in the existing literature. The ensuing results are compared and contrasted between the existing standard and the SDM multivariate approach. Finally, Section IV outlines the summary of the key results and draws the concluding remarks.

\section{II: State- Dependent Models: A Multivariate Framework Extension}

In this section we describe the general structure of the SDM's and the extended multivariate approach. We also consider the problem of identifying SDM's. We explain how from the fitted model we may obtain an over view of the non-linear structure of the model, which may lead us toward a more specific non-linear model. A more extensive discussion of these models is found in Priestley (1980) and an extensive study of the application of statedependent models to real and simulated data is given in Haggan et al (1984).

Consider the following linear $\mathrm{AR}(k)$ model

$$
Y_{t}+\phi_{1} Y_{t-1}+\ldots+\phi_{p} Y_{t-p}=\mu+\varepsilon_{\mathrm{t}}
$$

where $\left\{\varepsilon_{t}\right\}$ is a sequence of independent zero-mean random error terms and $\mu, \phi_{1}, \ldots, \phi_{p}$ are constants, then at time $t-1$ the future development of the process $\left\{Y_{t}\right\}$ is determined by the values $\left\{Y_{t-1}, \ldots, Y_{t-p}\right\}$, together with future values of $\left\{\varepsilon_{t}\right\}$. Hence, the vector: $y_{t-1}=\left\{Y_{t-1}, \ldots, Y_{t-p}\right\}^{\prime}$ may be regarded as the 'state-vector' of the process $\left\{Y_{t}\right\}$. That is, the only information in the 'past' of the process relevant to the future development of the process is contained in the state-vector. 
The SDM extends the idea of the linear AR time series model by allowing the coefficients of model (1) to become functions of the state-vector $y_{t-1}$, leading to the general non-linear model:

$$
Y_{t}+\phi_{1}\left(y_{t-1}\right) Y_{t-1}+\ldots . . \phi_{p}\left(y_{t-1}\right) Y_{t-p}=\mu\left(y_{t-1}\right)+\varepsilon_{\mathrm{t}}
$$

This model possess considerable degree of generality and, in fact, the SDM scheme does include the linear AR model and the main types of specific non-linear time series models if one employs particular forms of the coefficient $, \mu, \phi_{1}, \ldots, \phi_{p}$. (see Haggan et al, 1984).

In this section we will show how to extend the AR-SDM model (2) in to a general multivariate form to include exogenous variables. We will show this by having one explanatory variable in the model. However, these ideas may be readily extended to more than one explanatory variable.

If we extend the AR-SDM model (2) to include the explanatory variable $X_{t}$ we can write it in SDM form,

$$
\begin{aligned}
Y_{t}+\phi_{1}\left(y_{t-1}\right) Y_{t-1}+\ldots+\phi_{p}\left(y_{t-1}\right) Y_{t-p}= & \\
& \mu\left(y_{t-1}\right)+\theta_{0}\left(y_{t-1}\right) X_{t}+\theta_{1}\left(y_{t-1}\right) X_{t-1}+\ldots+\theta_{q}\left(y_{t-1}\right) X_{t-q}+\varepsilon_{\mathrm{t}}
\end{aligned}
$$

where the state vector at time $t-1$ is given by:

$$
y_{t-1}=\left\{X_{t-1}, \ldots, X_{t-q}, Y_{t-1}, \ldots, Y_{t-p}\right\}^{\prime}
$$

As can be seen, model (3) has additional parameters: $\theta_{0}, \theta_{1} \ldots \theta_{q}$ (parameter $\theta_{0}$ can be dropped from the model if the explanatory $X$ is not observed at time $t$ ). 
In fitting this generalized SDM model to any set of data, we are concerned with the estimation of the parameters $\mu, \theta_{0}, \theta_{1}, \ldots, \theta_{q}, \phi_{1}, \ldots, \phi_{p}$. However, these coefficients depend on the state vector $y_{t-1}$, and the estimation problem thus becomes the estimation of the functional form of this dependency. In order to estimate these coefficients, a recursive method similar to that of Harrison and Stevens (1976) is used. However, the basic difference between the two methods is that the Harrison-Stevens scheme employs a model with 'timedependent' coefficients (giving a non-stationary model), while in the SDM model the coefficients are 'state-dependent', making the model non-linear.

Priestly (1980) has shown it is possible to base the estimation procedure on the (extended) Kalman Filter algorithm provided some assumptions are made about the parameters. The simplest non-trivial assumption that can be made is that the parameters are linear functions of the state-vector $y_{t}$, so that for each $u$ :

$$
\begin{aligned}
& \phi_{u}\left(y_{t}\right)=\phi_{u}^{(0)}+y_{t}^{\prime} \cdot \gamma_{u} \\
& \theta_{u}\left(y_{t}\right)=\theta_{u}^{(0)}+y_{t}^{\prime} \cdot \beta_{u}
\end{aligned}
$$

We may adopt similar model for $\mu\left(y_{t}\right)$ as:

$$
\mu\left(y_{t}\right)=\mu^{(0)}+y_{t}^{\prime} \cdot \alpha
$$

where $\mu^{(0)}, \phi^{(0)}, \theta^{(0)}$ are constants, and $\alpha, \gamma_{u}, \beta_{u}$ are 'gradient' vectors. Although this assumption clearly cannot represent all types of non-linear model, it is reasonable to assume that these parameters may be represented locally as linear functions of $y_{t}$ (Priestley, 1980). This assumption is valid provided $\mu,\left\{\phi_{u}\right\}$ and $\left\{\theta_{u}\right\}$ are slowly changing functions of $y_{t}$. 
Based on these assumptions, 'updating' equations for the parameters may be written as follows:

$$
\begin{aligned}
& \mu\left(y_{t+1}\right)=\mu\left(y_{t}\right)+\Delta y_{t+1}^{\prime} \cdot \alpha^{(t+1)} \\
& \phi_{u}\left(y_{t+1}\right)=\phi_{u}\left(y_{t}\right)+\Delta y_{t+1}^{\prime} \cdot \gamma_{u}^{(t+1)} \\
& \theta_{u}\left(y_{t+1}\right)=\theta_{u}\left(y_{t}\right)+\Delta y_{t+1}^{\prime} \cdot \beta_{u}^{(t+1)}
\end{aligned}
$$

where $\Delta y_{t+1}=y_{t+1}-y_{t}$. The 'gradient' parameters: $\alpha^{(t)}, \beta_{0}^{(t)} \beta_{1}^{(t)}, \ldots, \beta_{q}^{(t)}, \gamma_{1}^{(t)}, \ldots, \gamma_{p}^{(t)}$ are unknowns to be estimated. The basic strategy is to allow these parameters to wander in the form of 'random walks'. The random walk model for the gradient parameters may be written in matrix form as

$$
\mathbf{B}_{\mathbf{t}+\mathbf{1}}=\mathbf{B}_{\mathbf{t}}+\mathbf{V}_{\mathbf{t}+\mathbf{1}}
$$

where $\mathbf{B}_{\mathbf{t}+\mathbf{1}}=\left(\alpha^{(t)}, \beta_{0}^{(t)}, \ldots . \beta_{q}^{(t)}, \gamma_{1}^{(t)}, \ldots . ., \gamma_{p}^{(t)}\right)^{\prime}$ and $\left\{\mathbf{V}_{\mathbf{t}}\right\}$ is a sequence of independent matrixvalued random variables such that $\mathbf{V}_{\mathbf{t}} \sim \mathbf{N}\left(0, \sum_{v}\right)$. The estimation procedure then determines, for each $t$, values of $\mathbf{B}_{\mathbf{t}+\mathbf{1}}$, which minimize the discrepancy between the observed value of $Y_{t+1}$ and its predictor, $\hat{Y}_{t+1}$ computed from the model fitted at time $t$.

The generalized SDM model (3) can be rewritten in a state-space form as respectively the observation and state equations:

$$
\begin{aligned}
& Y_{t}=H_{t} \psi_{t}+\varepsilon_{t} \\
& \psi_{t}=\mathbf{F}_{\mathbf{t}-\mathbf{1}} \psi_{t-1}+\mathbf{W}_{\mathbf{t}}
\end{aligned}
$$

where the state-vector $\psi_{t}$ is a vector of all current parameters of the model: 


$$
\psi_{t}=\left(\mu^{(t-1)}, \theta_{0}^{(t-1)}, \ldots, \theta_{q}^{(t-1)}, \phi_{1}^{(t-1)}, \ldots, \phi_{p}^{(t-1)}, \alpha^{(t)^{\prime}}, \beta_{0}^{(t)^{\prime}}, \ldots, \beta_{q}^{(t)^{\prime}}, \gamma_{0}^{(t)^{\prime}}, \ldots, \gamma_{p}^{(t)^{\prime}}\right)
$$

and $H_{t}$ and $\mathbf{W}_{t}$ are defined as:

$$
\begin{aligned}
& H_{t}=\left(1, X_{t}, X_{t-1}, \ldots X_{t-q},-Y_{t-1}, \ldots,-Y_{t-p}, 0, \ldots, 0\right)^{\prime} \\
& \mathbf{W}_{t}=\left(0,0, \ldots, 0, v_{1, t}^{\prime}, \ldots, v_{q+p+2, t}^{\prime}\right)^{\prime}
\end{aligned}
$$

$\mathbf{W}_{t}$ includes $p+q+2$ zero elements and $v_{1, t}^{\prime}, \ldots, v_{q+p+2, t}^{\prime}$ are the columns of the matrix $\mathbf{V}_{\mathbf{t}}$.

.The transition matrix $\mathbf{F}_{\mathbf{t}}$ is also defined as:

$$
\mathbf{F}_{\mathbf{t}-1}=\left[\begin{array}{cccc}
I_{p+q+2} & \Delta y_{t-1}^{\prime} \ldots \ldots . & 0 \\
& 0 & \ldots . \Delta y_{t-1}^{\prime} \\
& & I_{(p+q+1)(p+q+2)}
\end{array}\right]
$$

where $\Delta y_{t-1}=\left(X_{t-1}-X_{t-2}, \ldots . ., X_{t-q}-X_{t-q-1}, Y_{t-1}-Y_{t-2}, \ldots ., Y_{t-q}-Y_{t-p-1}\right)$

Applying the Kalman algorithm (Kalman, 1963) directly to the equations (5) and (6) gives the recursion

$$
\hat{\psi}_{(\mathbf{t})}=\mathbf{F}_{\mathbf{t}-1} \hat{\psi}_{(\mathbf{t}-1)}+\mathbf{K}_{\mathbf{t}}\left\{\mathbf{X}_{\mathbf{t}}-\left[\mathbf{H}_{\mathbf{t}} \cdot \mathbf{F}_{\mathbf{t}-\mathbf{1}} \cdot \hat{\psi}_{(\mathbf{t}-1)}\right]\right\}
$$

and $\mathbf{K}_{\mathbf{t}}$, the 'Kalman gain' matrix, is given by

$$
\mathbf{K}_{\mathbf{t}}=\boldsymbol{\Phi}_{\mathbf{t}}\left(\mathbf{H}_{\mathbf{t}}\right)^{\prime} \sigma_{e}^{-2}
$$

$\boldsymbol{\Phi}_{\mathbf{t}}$ being the variance-covariance matrix of the one-step prediction error of $\psi_{t}$, i.e., 


$$
\mathbf{\Phi}_{\mathbf{t}}=E\left[\left\{\psi_{t}-\mathbf{F}_{t-1} \cdot \hat{\psi}_{t-1}\right\}\left\{\psi_{t}-\mathbf{F}_{t-1} \cdot \hat{\psi}_{t-1}\right\}^{\prime}\right]
$$

and $\sigma_{e}^{2}$ is the variance of the one-step ahead prediction error of $\mathbf{Y}_{\mathbf{t}}$ i.e., $\sigma_{e}^{2}$ is the variance of $e_{\mathbf{t}}=\left\{\mathbf{Y}_{\mathbf{t}}-\left[\mathbf{H}_{\mathbf{t}} \cdot \mathbf{F}_{\mathbf{t}-1} \cdot \hat{\psi}_{\mathbf{t}-1}\right]\right\}$ or $e_{\mathbf{t}}=\left\{\mathbf{H}_{\mathbf{t}} \cdot\left[\psi_{t}-\mathbf{F}_{\mathbf{t}-1} \cdot \hat{\psi}_{\mathbf{t}-1}\right]\right\}+\varepsilon_{t}$, thus we can write $\sigma_{e}^{2}=\left[\mathbf{H}_{\mathbf{t}} \boldsymbol{\Phi}_{\mathbf{t}}\left(\mathbf{H}_{\mathbf{t}}\right)^{\prime}+\sigma_{\varepsilon}^{2}\right]$. If the variance-covariance matrix of $\left(\psi_{t}-\hat{\psi}_{t}\right)$ is denoted by $\mathbf{C}_{\mathbf{t}}$, then successive values of $\hat{\psi}_{t}$ may be estimated by using the standard recursive equations for the Kalman Filter, namely:

$$
\begin{aligned}
& \mathbf{K}_{\mathbf{t}}=\boldsymbol{\Phi}_{\mathbf{t}}\left(\mathbf{H}_{\mathbf{t}}\right)^{\prime}\left[\mathbf{H}_{\mathbf{t}} \boldsymbol{\Phi}_{\mathbf{t}}\left(\mathbf{H}_{\mathbf{t}}\right)^{\prime}+\sigma_{\varepsilon}^{2}\right]^{-1} \\
& \boldsymbol{\Phi}_{\mathbf{t}}=\mathbf{F}_{\mathbf{t}-\mathbf{1}} \mathbf{C}_{\mathbf{t}-\mathbf{1}}\left(\mathbf{F}_{t-1}\right)^{\prime}+\Sigma_{W} \\
& \mathbf{C}_{\mathbf{t}}=\boldsymbol{\Phi}_{\mathbf{t}}-\mathbf{K}_{\mathbf{t}}\left[\mathbf{H}_{\mathbf{t}} \boldsymbol{\Phi}_{\mathbf{t}}\left(\mathbf{H}_{\mathbf{t}}\right)^{\prime}+\sigma_{\varepsilon}^{2}\right] \mathbf{K}_{\mathbf{t}}^{\prime}
\end{aligned}
$$

where $\Sigma_{W}=\left(\begin{array}{cc}0 & 0 \\ 0 & \Sigma_{v}\end{array}\right)$

In practice, this recursive procedure must be started at some value of $t=t_{0}$, and hence initial values are required for $\hat{\psi}_{t_{0}-1}$ and $\hat{\mathbf{C}}_{t_{0}-1}$. Equation (3) represents a 'locally' linear model and in finding these initial values, we treat parameters in equation (3) as constant and apply the same procedure as Haggan et al (1984) as follows:

(i) Take an initial stretch of the data, say the first $2 m$ observations on $\left(Y_{t}, X_{t}\right)$, and fit a linear model. This will provide initial values $\hat{\mu}, \hat{\theta}_{0}, \ldots, \hat{\theta}_{q}, \hat{\phi}_{1}, \ldots, \hat{\phi}_{q}$ and the residual variance of the model, $\hat{\sigma}_{\varepsilon}^{2}$. 
(ii) Start the recursion midway along the initial stretch of data at $t_{o}=m$, (where it seems reasonable to assume that the initially estimated parameter values are most accurate),thus setting

$$
\begin{aligned}
& \hat{\psi}_{t_{0}-1}=\left(\hat{\mu}, \hat{\theta}_{0}, \hat{\theta}_{1}, \ldots, \hat{\theta}_{q}, \hat{\phi}_{1}, \ldots, \hat{\phi}_{p}\right)^{\prime} \\
& \hat{\mathbf{C}}_{t_{0}-1}=\left(\begin{array}{cc}
\hat{R}_{\mu, \theta, \phi} & 0 \\
0 & 0
\end{array}\right)
\end{aligned}
$$

where $\hat{R}_{\mu, \theta, \phi}$ is the estimated variance-covariance matrix of $\hat{\psi}_{t_{0}-1}=(\hat{\mu}$, $\left.\hat{\theta}_{0}, \hat{\theta}_{1}, \ldots, \hat{\theta}_{q}, \hat{\phi}_{1}, \ldots, \hat{\phi}_{p}\right)^{\prime}$ obtained from the initial linear model fitting. It also seems reasonable to set all the initial gradients to zero, assuming that the initial values are reasonably accurate at $t_{o}=m$. It remains to choose reasonable values for $\Sigma_{v}$, the variance-covariance matrix of $\mathbf{V}_{\mathbf{t}+\mathbf{1}}$ (and, hence by implication, to choose values for $\Sigma_{w}$ ). The choice of $\Sigma_{v}$ depends on the assumed 'smoothness' of the model parameter as functions of $y_{t}$. The diagonal elements of $\Sigma_{v}$ are set equal to $\hat{\sigma}_{\varepsilon}^{2}$ multiplied by a constant called the 'smoothing factor', and the off-diagonal elements are set equal to zero. However, if the elements of $\Sigma_{v}$ are set too large, the estimated parameters become unstable, but if the elements of $\Sigma_{v}$ are made too small, it is difficult to detect the non-linearity present in the data since the procedure is then virtually equivalent to the recursive fitting of a linear model.

The best procedure in practice appears to be to reduce the magnitude of the 'smoothing factor' until the parameters show stable behavior. If the parameters still appear to be far from 'smooth', the smoothing factor may be reduced further (Haggan et 
al, 1984). In addition, the parameters may be smoothed by a multi-dimensional form of the non-parametric function fitting technique (see for example Priestley and Chao, 1972). Having carried out this procedure, the resulting parameter surfaces can give a clearer idea of the type of non-linearity present in the model, and provide indications of the special type of non-linear dependency.

\section{III: An Application of the Multivariate SDM Approach: The Sentiment-Consumption Relationship}

Since Katona's (1968) seminal paper the role of the consumer sentiment index (henceforth CSI) in both predicting and understanding the causes of business cycles has been investigated widely. Recent examples are found in Barsky and Sims (2012), Starr (2012), and Nguyen and Claus (2013). They, however, come to a variety of conclusions ranging from CSI has little or no additional explanatory power about economic activity once the effects of "fundamental" variables are accounted for. Others stress that CSI embodies useful "animal spirits" and/or "news" information (see e.g. Golinelli and Parigi, 2004).

Regardless, in general, the existing literature has analyzed these issues in a linear context (with possible ad hoc shifts in parameters). Such an assumption broadly contradicts Katona's own views that CSI is influenced by psychological factors and are particularly pertinent during special events when households are more likely to change their attitude (Katona, 1977). In addition when such special events occur, the size of shocks to CSI matters, inducing non-linear responses.

The generalized SDM approach proposed in this paper enables us to explore the consumption-sentiment relationship over different states, without needing to assume ad hoc forms of non-linearity. Hence, following Inoue and Kilian (2004), we are able to assess the in-sample predictability of consumption with indicators (including the CSI). 
Equation (10) below specifies a linear reduced form relationship found in the existing literature (see e.g. Carroll et al, 1994, and Dees and Soares-Brinca, 2013). Equation (10) is estimated over an extended sample (including the Great Recession period) and is used as the benchmark model for our generalized SDM.

$$
\Delta \ln C_{t}+\sum_{i=1}^{5} \phi_{i} \Delta \ln C_{t-i}=\mu+\theta \ln C S I_{t-1}+\lambda \Delta \ln Y_{t-1}+\varepsilon_{t}
$$

where $C_{t}$ denotes real personal consumption expenditure while its lags measure the degree of stickiness in consumption growth either due to habits or inattention (Carroll et al, 2011). We specify on the left side of the equation both consumption and its lags to be consistent with the SDM notation outlined in Section II. CSI denotes consumer sentiment, $Y$ the real disposable personal income, and $\varepsilon$ is the unobservable error. ${ }^{1}$

If the $\log$ of CSI is significant, we reject the hypothesis that the CSI predicts consumption only through the income channel (i.e. it carries useful information besides that of expected income, see Carroll et al., 1994). Lagged income growth is included as a control variable (see Flavin, 1981, Campbell and Mankiw, 1989, and Bram and Ludvigson, 1998). Furthermore, as argued in Campbell and Mankiw (1989), the real disposable personal income can proxy the share of rule-of-thumb consumers (in addition to the life-cyclers/rational ones).

Table 1 reports the preliminary estimated results for the linear model (10) and some ad hoc non-linear extensions (see also model (11) below).

\section{Table 1 here}

\footnotetext{
${ }^{1}$ Details about data sources and definitions are in the Data Appendix.
} 
The OLS results are found in column (1) and the in-sample predictability of consumption using the CSI appears clear: CSI significantly leads consumption spending, in addition to income. $^{2}$

The residual diagnostics in column (1), nevertheless, raises a number of doubts about the data congruency of the linear specification. A number of issues emerge pertaining to heteroscedasticity, misspecification and structural breaks. An initial reaction to these caveats is to arbitrarily assume shifts in the impact of CSI on consumption during the Great Moderation of 1984-2007 (henceforth GM). Columns (2) and (3) report the estimates of equation (10) in two sub-periods: one excluding the GM period and another just the GM period. The misspecification problems are partially dealt with and also there is some support for parameter shifts as a viable means to improve the data congruency of the linear model.

The estimates of the income effect ( $\lambda$ ) are quite stable over the two subsamples: it is only slightly higher in the GM period, when the Campbell-Mankiw's rule of thumb to consume is probably less costly. Conversely, the largest shift is the autoregressive dynamics. The lagged consumption growth passes from moderate overshooting (when the GM period is excluded) to moderate persistence (when the focus is just on the GM period). As noted earlier, less costly inattentiveness makes the occurrence of habit-forming behavior during the GM period more likely. The estimated sentiment effect $(\theta)$ when the GM period is excluded (see column (2)) is considerably higher than in the GM period (column (3)). The insignificant sentiment clearly suggests that CSI explains consumption only when a number of highly variable shocks occur. Hence, the rejection of $\theta=0$ over the entire sample period (column (1)) conceals breaks in $\theta$ over time and/or across states, leading to biased estimates.

\footnotetext{
${ }^{2}$ This result is also robust with the inclusion of a number of financial indicators such as the log-change in the stock price or the Fed Funds rate (see Leeper,1992, Bram and Ludvigson, 1998, Ludvigson, 2004, and Croushore, 2005). So the CSIconsumption correlation is also not merely driven by the financial indicators. Details are available from the authors upon request.
} 
As acknowledged by Inoue and Kilian (2004), an overall rejection could dissemble a variety of scenarios. Likewise, as suggested by Katona, the CSI parameter is expected to be significant in some periods (i.e. when the GM period is excluded) and not in other periods (i.e. over the GM). Overall, the results in the first three columns of Table 1 suggest that parameters of the linear model can be different for each of the sub-sample (which is assumed to be known a-priori) and can also partly account for the statistical problems. However, at least two issues remain unresolved: (i) the arbitrary selection of the "relevant" sub-periods; and (ii) the inefficient estimates of many parameters, as their number in the linear model is multiplied by the number of subsamples in which the span is divided.

Although still arbitrary, an alternative is to focus on the impact of business cycles on the sentiment parameter $\theta$. Column (4) reports the estimated parameters of an ad hoc nonlinear extension of model (10) focusing only on CSI:

$$
\Delta \ln C_{t}+\sum_{i=1}^{5} \phi_{i} \Delta \ln C_{t-i}=\mu+\left(\theta+\delta I_{t}\right) \ln C S I_{t-1}+\lambda \Delta \ln Y_{t-1}+\varepsilon_{t}
$$

where the shifts $(\delta)$ in $\theta$ are determined by the Heaviside indicator $I_{t}$ such that $I_{t}=1$ during the periods in which the economy downturns on the basis of the NBER dating of the cycle (see Data Appendix).

Despite evidence of heteroscedasticity and breaks, $R^{2}$ increases considerably from 0.24 to 0.35 (see columns (1) and (4)), suggesting that allowing for a non-linear CSIconsumption relationship improves the explanation of consumption growth. In particular, the impact of CSI on consumption growth during the downturn phases, as measured by $\theta+\delta$, goes down about $20 \%$ due to a significant negative $\delta$ estimate. The latter suggests the likelihood of a smaller CSI impact during downturns. This result, however, is contrary to the "classical" prediction that CSI affects consumption growth greater during recessions. 
Alternatively, "special events" can also be accounted for in equation (11) by (arbitrarily) defining $I_{t}=1$ if $\left|\Delta \ln C S I_{t-1}\right| \geq \tau$ and $I_{t}=0$ if $\left|\Delta \ln C S I_{t-1}\right|<\tau$, where $\tau=k \times \sigma$, and where $k$ sets the amplitude of the range of the "extreme" cases, and $\sigma$ is the standard deviation of CSI quarterly growth (0.064 in our case). The results outlined in column (5) indicates a positive $5-6 \%$ shift in the magnitude of $\delta$ when CSI growth is outside the $\pm 2 \sigma$ interval $^{3}$, indicating that extreme CSI shocks has a larger impact on consumption. ${ }^{4}$. Importantly, this stresses the risk of making ad hoc assumptions about Katona's "special events". Likewise for the inferences and forecasts when we extend equation (10) to account for non-linearity. ${ }^{5}$ For example, equation (11) may overcome the inefficiency issue but only after introducing breaks arbitrarily, albeit based on a priori knowledge.

Such difficulties arise in the main because we always model data starting from specific (linear) to general (non-linear) models; i.e. we extend equation (10) to (11) without any clear idea about how to implement such extensions. We simply assume in each case that the provided solutions are admissible. The SDM approach, on the other hand, can deliver a number of stylized facts regarding the consumption-sentiment relationship without resorting to ad hoc extensions, as it begins with a general specification. However, the SDM outcomes below are still subject to the well-known objections for any reduced form formulation. Therefore, we must view them as suggestive rather than evidence about well formulated hypotheses.

\footnotetext{
${ }^{3}$ In this case, $I_{t}=1$ marks 16 observations, i.e. about the $5 \%$ of the sample.

${ }^{4}$ Although their $I_{t}$ indicator is defined in a similar way as here, our outcome varies with Dees and Soares-Brinca (2013) because they assume that the CSI effect on consumption only works when CSI growth rate is outside the interval, i.e. that $\theta=0$ in model (11). The significant estimate of $\theta$ in column (5) rejects their assumption in our context.

${ }^{5}$ If we refer to the three cases analysed in columns (2)-(5) of Table 1, in columns (2)-(3) the ad hoc non-linearity assumes that all parameters of model (10) shift during the GM phase, while those in columns (4)-(5) assume that only the CSI parameter shifts following the NBER cycle, or when the CSI growth is larger than two CSI standard deviations.
} 
In the reminder of this section, we focus on the consumption-sentiment relationship using a parsimonious SDM where the dynamics of past consumption is restricted to lag $5 .{ }^{6}$

$$
\Delta \ln C_{t}+\phi\left(y_{t-1}\right) \Delta \ln C_{t-5}=\mu\left(y_{t-1}\right)+\theta\left(y_{t-1}\right) \ln C S I_{t-1}+\lambda\left(y_{t-1}\right) \Delta \ln Y_{t-1}+\varepsilon_{t}
$$

where $y_{t-1}=\left\{\ln C S I_{t-1}, \Delta \ln Y_{t-1}, \Delta \ln C_{t-5}\right\}$. The state representation of CSI enables a varying effect on consumption in specific phases of the cycle and likewise for all the other explanatory variables. Therefore SDM can relax the need for ad hoc assumptions as before because it allows for state-dependent effects to all the variables of interest (and not merely to CSI). Finally, it must be emphasized that the SDM algorithm operates purely on the data, without any prior knowledge about the underlying model.

In the first instance a linear model is first fitted to the first stretch of the data ${ }^{7}$, then the parameters of model (12) are estimated as described in Section II, using the recursion a smoothing factor in the range of $10^{-2}$ to $10^{-5}$ as suggested by Haggan et al(1984). The time patterns of the estimates of $\hat{\phi}, \hat{\theta}$ and $\hat{\lambda}$ are plotted in Figure 1. Figure 2 plots the estimates of $\hat{\theta}$ against the state vector (i.e. the variables CSI, growth in income and consumption), and also $\hat{\lambda}$ against the growth in income variable.

The time-varying effects of the explanatory variables on consumption spending are depicted in the four panels in Figure 1. The panels shows the impact of CSI (in panel $I$ with

\footnotetext{
${ }^{6}$ For the sake of robustness, we also tried adding single lags, or all the first 5 lags together. In all these experiments, lag 5 always proved to be the most relevant, and results were not sensitive to specific lag selection.

${ }^{7}$ The preliminary estimates over the first period of the data were: $\hat{\mu}=-0.0137, \hat{\phi}=0.252, \hat{\theta}=0.005, \hat{\lambda}=0.391$, $\hat{\sigma}_{\varepsilon}^{2}=0.00004$, and $\hat{R}=\left[\begin{array}{rrrr}0.0017 & -0.0008 & -0.0004 & 0.0014 \\ -0.0008 & 0.0087 & 0.00002 & -0.0015 \\ 0.0004 & 0.0002 & 0.0008 & -0.0003 \\ 0.0014 & -0.0015 & -0.0003 & 0.0125\end{array}\right]$
} 
shaded areas corresponding to the downturn phases of the NBER dating; and in panel $I I$ with the shaded $95 \%$ confidence interval), of income (panel $I I I$ ), and of the consumption lag 5 (panel $I V)$.

\section{Figure 1 here}

The estimated CSI-consumption relationship, depicted in panels $I$ and II, suggests four main outcomes. Firstly, the $95 \%$ confidence interval of the CSI parameter includes zero for all periods. The time-varying CSI parameter always explains consumption behavior together with income and, consequently, it is clearly an additional explanatory variable of consumption behavior (and not merely a proxy to predict income). Secondly, at the beginning of each recession phase the CSI effect on consumption increases rapidly and sharply. Thereby reinforcing CSI as a good predictor of consumption behavior at the beginning of an economic slowdown (the only exception to this rule being the beginning of the very short recession period: 2001q2-2001q4). Thirdly, larger shifts in the CSI parameter occurs during the recessions in the 1970s. Similarly, at the beginning of the Great Recession of 2008q2$2009 q 2$ there is a clear increase in the CSI effect but, nevertheless, smaller than those found during the 1970s. Finally, during the recovery phases the CSI parameter decreases from the levels of the previous recession. These decreases are much smoother and only seldom revert to the pre-crisis level (for example, in the current period the CSI parameter is not significantly lower than that estimated at the beginning of the Great Recession).

Campbell and Mankiw (1989) argued that current income change affects consumption if a proportion of households follow a simple rule-of-thumb. A prevailing criticism of this proposition is they assume a fixed income parameter (see Ando (1989)). This assumption is relaxed here and using the SDM approach the parameter is allowed to be state-varying. The time-varying parameter is plotted in panel III of Figure 1 . There is a steady fall suggesting a reduction in the proportion of the rule-of-thumb households starting at the 
beginning of the GM. ${ }^{8}$ However, the $95 \%$ confidence interval of the income effect suggests that the random walk model of consumption advocated by Hall (1978) is rejected even during the period of GM. Finally, the varying effect of past changes of consumption is reported in panel $I V$. This emphasizes its significance which, in turn, reinforces the rejection of the Hall (1978) model suggesting consumption stickiness and habits persistence (see also Carroll et al, 1994). The overshooting of the steady state of consumption growth has been fairly moderate in mid-1970s and lie substantially within the same confidence interval.

The most relevant feature of SDM is that it allows the impact on consumption changes to vary with the state of the explanatory variables. The four panels in Figure 2 report the estimated non-linear CSI parameter (horizontal axis) against selected states of the explanatory variables (vertical axis). Panels $I$ and $I I$ show how the impact of CSI on consumption spending evolves with respect to the states of CSI levels and quarterly growth rate of income respectively. Panel III focuses on the same CSI effect but with respect to consumption lags, and panel $I V$ shows the effect of income on consumption with respect to quarterly income growth ${ }^{9}$.

\section{Figure 2 here}

Panel $I$ in Figure 2 indicates that the estimated CSI parameter, which depends on the CSI level, depicts an ESTAR function. The estimated parameter reaches its lowest point when the level of CSI is 100, i.e. when households' sentiment is indifferent. However, Panel II clearly shows that the CSI effect on consumption is an LSTAR function. It supports the view that positive changes in income are associated with smaller confidence impact on consumption. So when income growth is negative (symptomatic of "bad news") the CSI

\footnotetext{
${ }^{8}$ During the GM period uncertainty reduced considerably resulting in more households behaving consistently with rational expectations.

${ }^{9}$ The parameters are smoothed using a non-parameter function fitting technique which employs a rectangular smoothing kernel.
} 
parameter proximate its highest values (around 0.025 ). On the other hand, when income growth is positive it drops (by about 20\%) to its lowest level. A similar LSTAR pattern is found in panel III, where the CSI effect on consumption is depicted with respect to past consumption growth rates. At first it reaches the highest level when the growth is negative, subsequently, decreasing smoothly when growth is between zero and $2 \%$. It reaches its minimum level when the growth is above $2 \%$. Habit formation is stronger when past consumption decreases (akin to asymmetric smoothing).

Finally, panel $I V$ in Figure 2 clearly indicates that the income effect on consumption (interpreted as the proportion of non-rational household) against the states of income growth follows an asymmetric path, specifically a LSTAR function. It is between $0.18-0.20$ for income growth below $0.5 \%$ (or negative), while it reaches higher levels (around 0.25 ) when the growth of income is substantial (i.e. higher than 3\%). More research is needed to interpret such asymmetry evidence with a structural model.

\section{IV: Summary and Concluding Remarks:}

The purpose of this paper is twofold. In the first instances, we develop a generalized State-Dependent Model (SDM) in a multivariate framework. This enables a multivariate approach to analyzing a general form of non-linearity which can be fitted without any specific prior assumption about the form of non-linearity. Secondly, the extended framework enables greater applicability for the empirical analysis of economic relationships and models. The paper significantly extends the existing literature on SDM and its applications where the focus has been on univariate analysis using a pure AR time series.

The present paper also provides an example by investigating empirically the relationship between households' income, sentiment and their consumption behavior. The role of the consumer sentiment both in predicting and understanding the causes of business 
cycles is an important one, and the existing literature has been largely analyzed in the linear context (with possible ad hoc shifts in parameters). Using the extended generalized SDM approach we show the significant non-linear effect of sentiment on consumption behavior, while also providing additional explanatory powers than mere proxies for income changes. 


\section{Data Appendix}

The variables of interest where sourced through Federal Reserve Economic Data (FRED). Details are in Table A.1, models' variables are plotted in Figure A.1. Data are quarterly covering the period 1953-2015. Monthly (CSI) data are converted in quarters by averaging. All models' variables are found to be stationary, see the lower panel of Table A.1.

Table A.1 here

Figure A.1 here 


\section{References}

Ando, A. (1989), "Comment", O. Blanchard and S. Fischer (eds.), NBER Macroeconomic Annual, Cambridge, MIT Press, pp. 234-244.

Barsky, R. B. and E. R. Sims (2012), "Information, animal spirits, and the meaning of innovations in consumer confidence", The American Economic Review, Vol. 102, No. 4, pp. 1343-1377.

Bram, J. and S. C. Ludvigson (1998), "Does consumer confidence forecast households expenditure? A sentiment index horse race", FRBNY Economic Policy Review, June, pp. 59-78.

Campbell, J. Y. and G. Mankiw (1989), "Consumption, income and interest rates: reinterpreting the time series evidence", O. Blanchard and S. Fischer (eds.), NBER Macroeconomic Annual, Cambridge, MIT Press, pp. 185-216.

Carroll, C., J. C. Fuhrer, D. W. Wilcox (1994), "Does consumer sentiment forecast households spending? If so, why?", The American Economic Review, Vol. 84, No. 5, pp. 1397-1408.

Carroll, C., M. Otsuka and J. Slacalek (2011), "How large are housing and financial wealth effects? A new approach", Journal of Money, Credit and Banking, Vol. 43, No. 1, pp. $55-79$.

Christiano, L. J. (1989), "Comment", O. Blanchard and S. Fischer (eds.), NBER Macroeconomic Annual, Cambridge, MIT Press, pp. 216-234.

Croushore, D. (2005), "Do consumer confidence indexes help forecast consumer spending in real time?", North American Journal of Economics and Finance, Vol. 16, pp. 435460.

Dees, S. and P. Soares-Brinca (2013), "Consumer confidence as a predictor of consumption spending: evidence for the United States and the Euro area", International Economics, Vol. 134, pp. 1-14.

Elliott, G. T., J. Rothemberg, and J. H. Stock (1996), "Efficient tests for an autoregressive unit root", Econometrica, Vol. 64, No. 4, pp. 813-836.

Flavin, M. (1981), "The adjustment of consumption to changing expectations about future income", Journal of Political Economy, Vol. 89, No. 5, pp. 974-1009.

Golinelli, R. and G. Parigi (2004), "Consumer sentiment and economic activity: a cross country comparison", Journal of Business Cycle Measurement and Analysis, Vol. 1, No. 2, pp. 147-170.

Haggan, V , S. M. Heravi and M. B. Priestley (1984), "A study of the application of statedependent models in non-linear time series analysis", Journal of Time Series Analysis, Vol. 5, No. 2, pp. 69-102.

Hall, R. (1978), "Stochastic implications of the life cycle - permanent income hypothesis: theory and evidence", Journal of Political Economy, Vol. 86, No. 6, pp. 971-987.

Harrison, P. J. and C. F. Stevens (1976), "Bayesian forecasting (with discussion)", Journal of the Royal Statistical Society Series B, Vol. 38, pp. 205-247. 
Inoue, A. and L. Kilian (2004), "In-sample or out-of-sample tests of predictability: which one should we use?", Econometric Reviews, Vol. 23, No. 4, pp. 371-402.

Kalman, R. A (1963), "Mathematical descriptions of linear dynamical systems", Journal of the Society for Industrial and Applied Mathematics Series A Control, Vol. 1, pp. 152192.

Katona, G. (1968), "Consumer behavior: theory and findings on expectations and aspirations", The American Economic Review, Vol. 58, No. 2, pp. 19-30.

Katona, G. (1977), Psychological Economics, New York, Elsevier.

Leeper, E. M. (1992), "Consumer attitudes: king for a day", FRB Atlanta Economic Review, July/August, pp. 1-15.

Ludvigson, S. C. (2004), "Consumer confidence and consumer spending", Journal of Economic Perspectives, Vol. 18, No. 2, pp. 29-50.

$\mathrm{Ng}$, S. and P. Perron (2001), "Lag length selection and the construction of unit root tests with good size and power", Econometrica, Vol. 69, No. 6, pp. 1519-1554.

Nguyen, V. H. and E. Claus (2013), "Good news, bad news, consumer sentiment and consumption behavior", Journal of Economic Psychology, Vol. 39, pp. 426-438.

Priestley, M. B. (1980), "State-dependent models: a general approach to non-linear time series analysis", Journal of Time Series Analysis, Vol. 1, No. 1, pp. 47-71.

Priestley, M. B. and M. T. Chao (1972), "Non-parametric function fitting", Journal of the Royal Statistical Society Series B, Vol. 43, pp. 244-255.

Starr, M. A. (2012), "Consumption, sentiment, and economic news", Economic Inquiry, Vol. 50, No. 4, pp. 1097-1111.

White, H. (1980), "A heteroskedasticity-consistent covariance matrix and a direct test for heteroskedasticity", Econometrica, Vol. 48, No. 4, pp. 817-838. 
Tab. 1 - The consumption-sentiment relationship in the linear/breaking context
Experiments $^{a}$
(1)
(2)
(3)
(4)

(5)

OLS estimates $^{b}$

\begin{tabular}{|c|c|c|c|c|c|c|c|c|c|c|}
\hline $\begin{array}{l}\text { Constant } \\
\text { Std error }\end{array}$ & $\begin{array}{r}\mathbf{- 0 . 0 6 9 1} \\
0.0191\end{array}$ & $* * *$ & $\begin{array}{r}-\mathbf{0 . 0 8 0 8} \\
0.0217\end{array}$ & $* * *$ & $\begin{array}{r}-\mathbf{0 . 0 2 3 2} \\
0.0231\end{array}$ & & $\begin{array}{r}\mathbf{- 0 . 0 3 9 5} \\
0.0145\end{array}$ & **** & $\begin{array}{r}\mathbf{- 0 . 0 6 5 3} \\
0.0169\end{array}$ & ***** \\
\hline $\begin{array}{l}\text { Sentiment effect } \\
\text { Std error }\end{array}$ & $\begin{array}{r}\mathbf{0 . 0 1 7 4} \\
0.0044\end{array}$ & $* * *$ & $\begin{array}{r}\mathbf{0 . 0 2 0 2} \\
0.0049\end{array}$ & $* * *$ & $\begin{array}{r}\mathbf{0 . 0 0 6 2} \\
0.0052\end{array}$ & & $\begin{array}{r}\mathbf{0 . 0 1 1 1} \\
0.0033\end{array}$ & $* * *$ & $\begin{array}{r}\mathbf{0 . 0 1 6 2} \\
0.0039\end{array}$ & **** \\
\hline $\begin{array}{l}\text { Shift in sentiment } \\
\text { Std error }\end{array}$ & & & & & & & $\begin{array}{r}\mathbf{- 0 . 0 0 1 9} \\
0.0003\end{array}$ & $* * *$ & $\begin{array}{r}\mathbf{0 . 0 0 0 9} \\
0.0004\end{array}$ & $* *$ \\
\hline $\begin{array}{l}\text { Income effect } \\
\text { Std error }\end{array}$ & $\begin{array}{r}\mathbf{0 . 1 8 9 9} \\
0.0503\end{array}$ & $* * *$ & $\begin{array}{r}\mathbf{0 . 1 3 6 9} \\
0.0611\end{array}$ & ** & $\begin{array}{r}\mathbf{0 . 1 6 6 6} \\
0.0657\end{array}$ & $* *$ & $\begin{array}{r}\mathbf{0 . 1 4 9 2} \\
0.0434\end{array}$ & $* * *$ & $\begin{array}{r}\mathbf{0 . 1 5 0 8} \\
0.0501\end{array}$ & $* * * *$ \\
\hline $\begin{array}{l}\text { Dynamics (sum of lags) }{ }^{c} \\
\text { Std error }\end{array}$ & $\begin{array}{r}\mathbf{0 . 1 9 7 5} \\
0.0888\end{array}$ & ** & $\begin{array}{r}\mathbf{0 . 1 9 0 3} \\
0.0732\end{array}$ & ** & $\begin{array}{r}-\mathbf{0 . 2 7 5 0} \\
0.0971\end{array}$ & $* * *$ & $\begin{array}{r}\mathbf{0 . 2 1 4 2} \\
0.0754\end{array}$ & $* * *$ & $\begin{array}{r}\mathbf{0 . 0 2 6 1} \\
0.1216\end{array}$ & \\
\hline Dynamics reduction $(P-v a l)^{c}$ & 0.2376 & & 0.4131 & & 0.9775 & & 0.4008 & & 0.8465 & \\
\hline Observations, $T$ & 249 & & 153 & & 96 & & 249 & & 249 & \\
\hline$R^{2}$ & 0.2438 & & 0.2726 & & 0.2217 & & 0.3507 & & 0.2664 & \\
\hline Std. error of the regression & 0.0061 & & 0.0070 & & 0.0043 & & 0.0057 & & 0.0061 & \\
\hline \multicolumn{11}{|l|}{ Misspecification tests (P-values) } \\
\hline - Autocorrelation & 0.0978 & & 0.6912 & & 0.8763 & & 0.0140 & & 0.1693 & \\
\hline - Heteroskedasticity & 0.0073 & & 0.0356 & & 0.1972 & & 0.0001 & & 0.0045 & \\
\hline - $\mathrm{ARCH}$ & 0.0677 & & 0.1127 & & 0.6982 & & 0.1510 & & 0.1593 & \\
\hline - Ramsey RESET & 0.0321 & & 0.0125 & & 0.8579 & & 0.3075 & & 0.0970 & \\
\hline $\begin{array}{l}\text { - Andrews max-F } \\
\text { (date of the break) }\end{array}$ & $\begin{array}{r}0.0259 \\
(1965 q 1)\end{array}$ & & 0.2663 & & 0.4649 & & & & & \\
\hline
\end{tabular}

( $\left.{ }^{a}\right)$ Columns' legend

(1) Linear model (10) over the whole period 1953Q1-2015Q1.

(2) Linear model (10) over the period 1953Q1-1983Q4 and 2008Q1-2015Q1 (i.e. excluding the Great Moderation).

(3) Linear model (10) over the period 1984Q1-2007Q4 (i.e. the Great Moderation period)

(4) Breaking model (11) over the whole period 1953Q1-2015Q1, with $I_{t}=1$ during the downturns of the NBER dating.

(5) Breaking model (11) over the whole period 1953Q1-2015Q1, with $I_{t}=1$ if $\left|\Delta \ln C S I_{t-1}\right| \geq 2$ times the sample std. deviation of $\Delta \operatorname{lnCSI}_{\mathrm{t}-1}$.

$\left({ }^{b}\right)$ When heteroskedasticity is detected, standard errors (below parameter estimates) are those of White (1980). ${ }^{* * *}$ and ${ }^{* * * *}$ means significant at 10,5 , and $1 \%$.

$\left(^{c}\right)$ Estimate of $\sum_{i=1}^{5} \phi_{i}$ in equations (10) and (11) after dynamics' reduction: in order to obtain a parsimonious model, we started from a fifth-order dynamics and restricted to zero those not significant lags (accordingly, the "Dynamics reduction" row reports the corresponding joint zero restrictions P-values). 
Tab. A.1 - Data source, definition and unit root tests

\begin{tabular}{|c|c|c|c|c|}
\hline Label & \multicolumn{2}{|l|}{ Definition } & \multicolumn{2}{|c|}{$\begin{array}{l}\text { Source: FRED (Economic Research, Federal } \\
\text { Reserve Bank of St. Louis) } \\
\text { https://research.stlouisfed.org/fred } 2 /\end{array}$} \\
\hline$C_{t}$ & \multicolumn{2}{|c|}{ Real Disposable Personal Income } & \multicolumn{2}{|c|}{$\begin{array}{l}\text { PCECC } 96 \text {, Billions of Chained } 2009 \text { Dollars, } \\
\text { Quarterly, Seasonally Adjusted Annual Rate. }\end{array}$} \\
\hline$C S I_{t}$ & \multicolumn{2}{|l|}{ Consumer Sentiment Index } & \multicolumn{2}{|c|}{$\begin{array}{l}\text { UMCSENT, Thomson Reuters-University of } \\
\text { Michigan: Consumer Sentiment. Index } \\
\text { 1966:Q1=100, Not Seasonally Adjusted. } \\
\text { Discontinued (usually mid-quarter month) from } \\
\text { 1952-11 to 1977-11, then monthly from 1978-1. }\end{array}$} \\
\hline$Y_{t}$ & \multicolumn{2}{|c|}{ Real Disposable Personal Income } & \multicolumn{2}{|c|}{$\begin{array}{l}\text { DPIC } 96, \text { Billions of Chained } 2009 \text { Dollars, } \\
\text { Quarterly, Seasonally Adjusted Annual Rate. }\end{array}$} \\
\hline \multirow[t]{2}{*}{$\begin{array}{l}\text { NBER } \\
\text { dating }\end{array}$} & \multicolumn{2}{|c|}{$\begin{array}{l}\text { US Business Cycle Expansions } \\
\text { and Contractions }\end{array}$} & \multicolumn{2}{|c|}{$\begin{array}{l}\text { Source: NBER's Business Cycle Dating } \\
\text { Committee. http://www.nber.org/cycles.html }\end{array}$} \\
\hline & $\begin{array}{l}\text { DF-GLS test statistic } \\
\text { Elliott et al (1996) }{ }^{a}\end{array}$ & \multicolumn{3}{|c|}{ Critical values } \\
\hline$\Delta \ln C_{t}$ & $-2.859^{* * *}$ & -2.574 & -1.942 & -1.615 \\
\hline $\ln C S I_{t}$ & $-3.298^{* * * *}$ & -2.574 & -1.942 & -1.615 \\
\hline$\Delta \ln Y_{t}$ & $-2.277^{* *}$ & -2.574 & -1.942 & -1.615 \\
\hline
\end{tabular}

$\left.{ }^{(a}\right)$ Sample period: 1953Q1-2015Q1. Test with intercept and automatic lag length selection based on Modified AIC (Ng and Perron, 2001). 
Fig. 1 - The effect on consumption spending over time of sentiment (CSI), income and dynamics
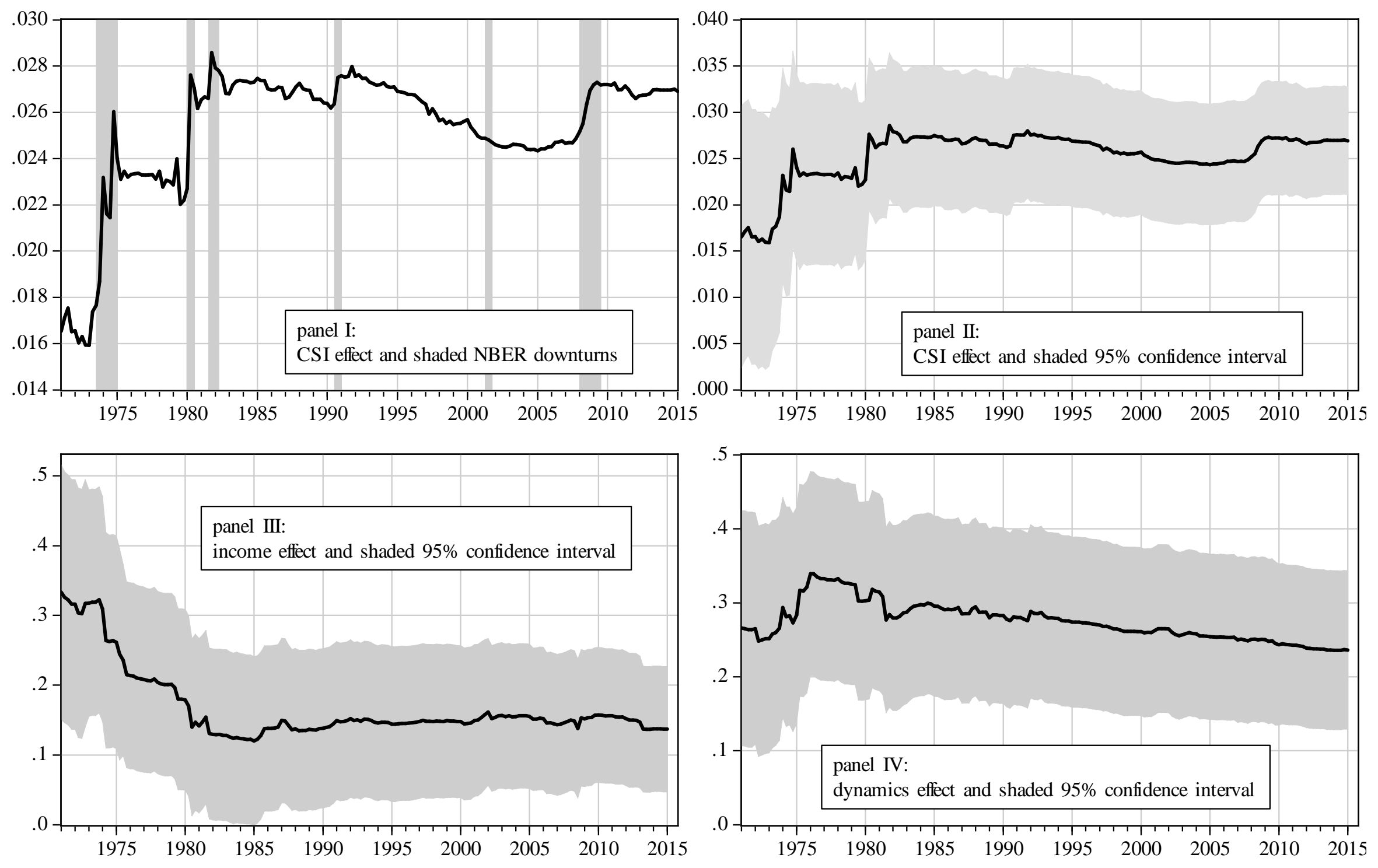
Fig. 2 - The effects on consumption spending over states of sentiment levels (CSI), and of income and consumption lags growth
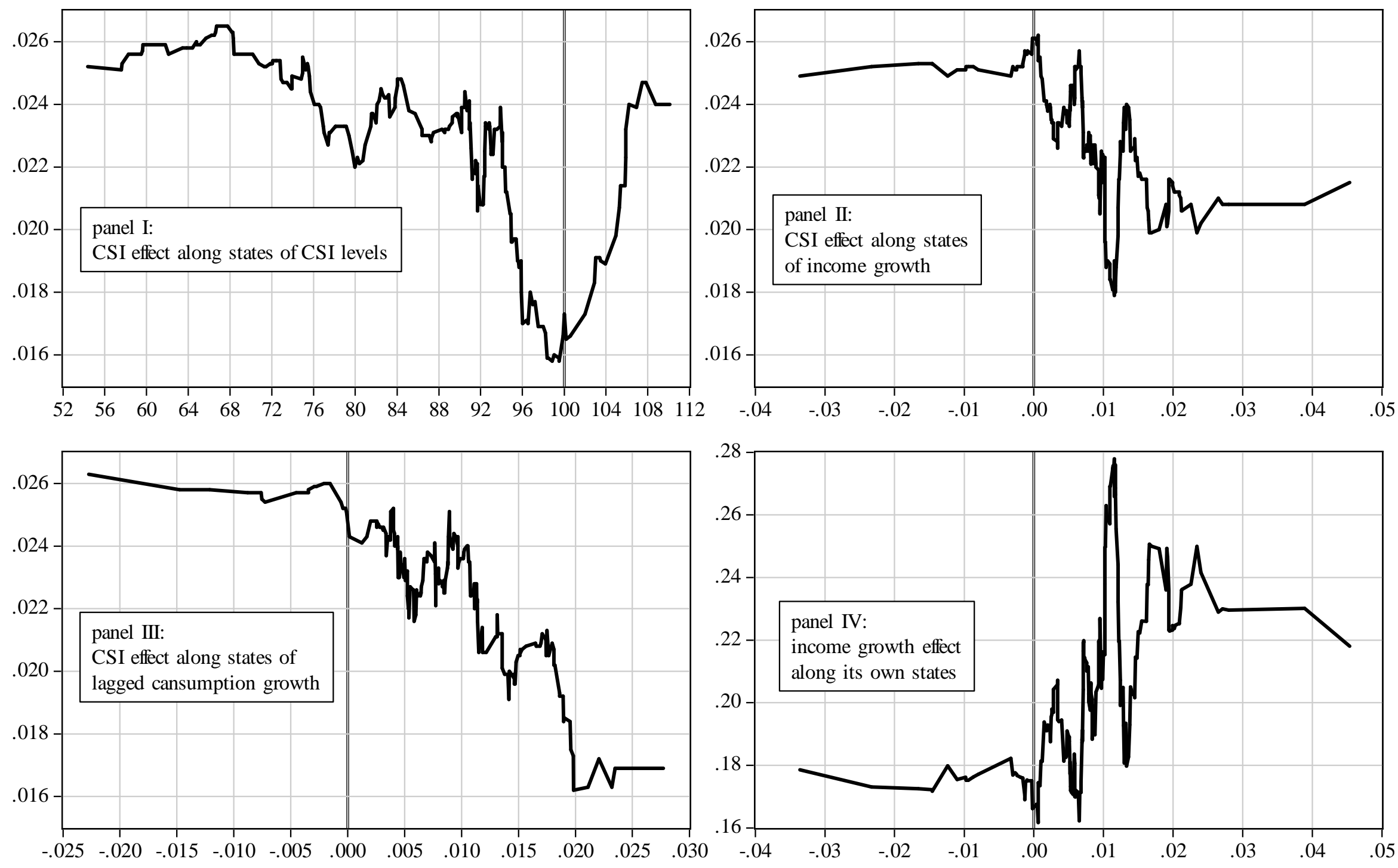
Fig. A.1 - The historical (1953-2015) pattern of the variables of interest

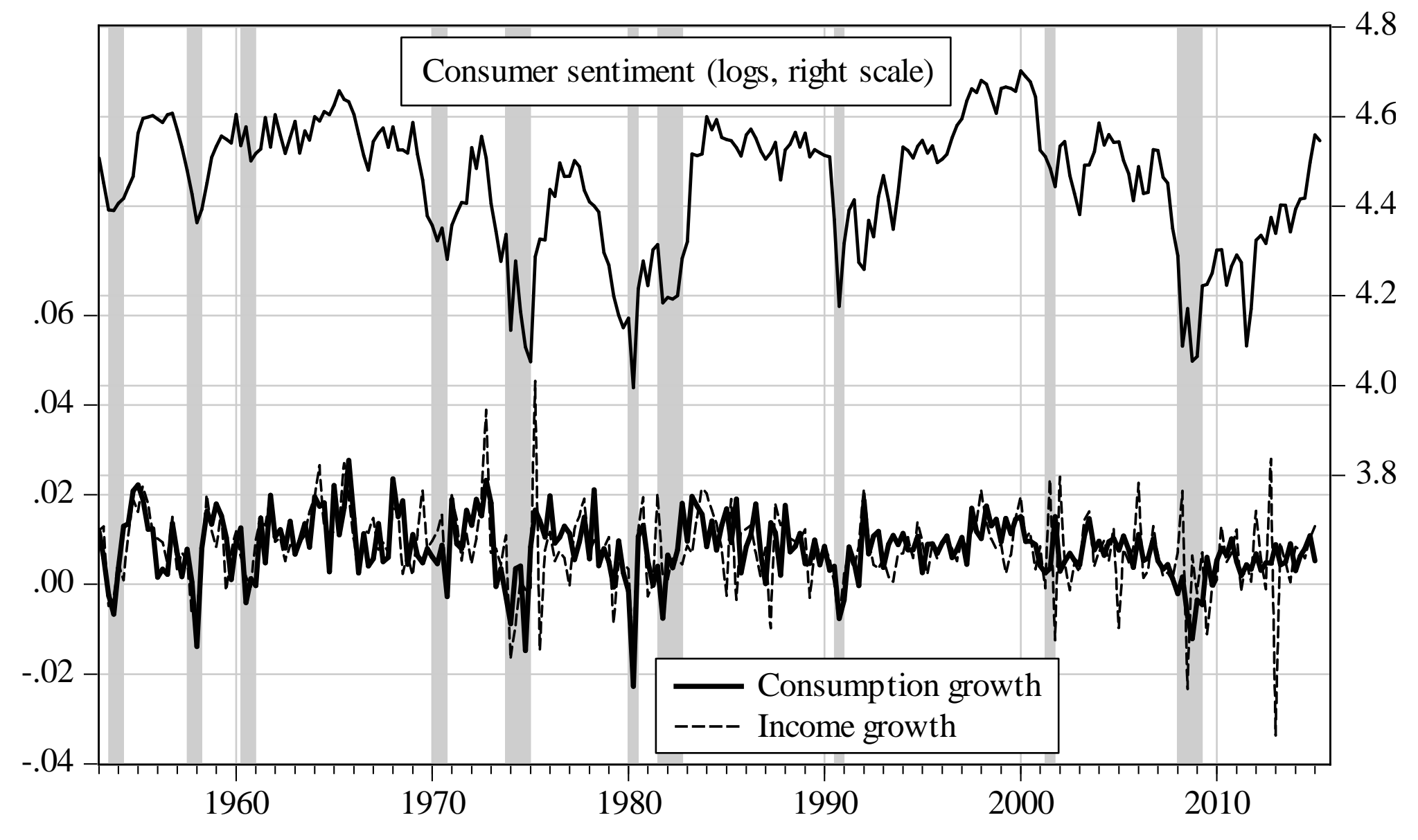




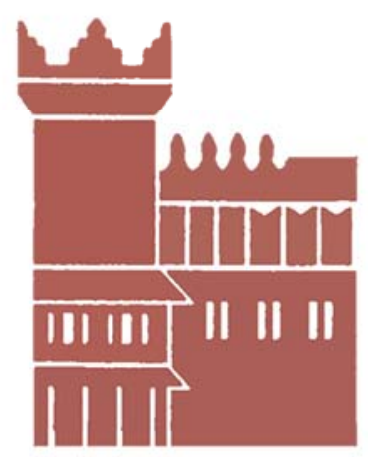

Alma Mater Studiorum - Università di Bologna DEPARTMENT OF ECONOMICS

Strada Maggiore 45

40125 Bologna - Italy

Tel. +39051 2092604

Fax +390512092664

http://www.dse.unibo.it 